\title{
Padrões de consumo de álcool e fatores associados entre adultos usuários de serviço de atenção básica do Rio de Janeiro, RJ, Brasil
}

\author{
Patterns of alcohol consumption and associated factors among adult \\ users of primary health care services of Rio de Janeiro, Brazil
}

Rafael Tavares Jomar ${ }^{1}$

Ângela Maria Mendes Abreu ${ }^{2}$

Rosane Harter Griep ${ }^{3}$

${ }^{1}$ Hospital do Câncer I, Instituto Nacional de Câncer. Pç. Cruz Vermelha 23/10, Centro. 20.230-130

Rio de Janeiro RJ Brasil. rafaeljomar@yahoo.com.br

${ }^{2}$ Departamento de

Enfermagem e Saúde

Pública, Escola de Enfermagem Anna Nery, Universidade Federal do Rio de Janeiro.

${ }^{3}$ Departamento de Biologia, Instituto Oswaldo Cruz, Fundação Oswaldo Cruz.
Abstract This study sought to verify the patterns of alcohol consumption and associated factors among adult users of a primary health care service in the city of Rio de Janeiro, Brazil. A household survey with a sample of 301 individuals who responded to a collection instrument containing the Alcohol Use Disorders Identification Test and social, demographic and health variables was conducted. Logistic regression was used to assess the association between the pattern of problematic alcohol consumption and the independent variables. Patterns of problematic alcohol consumption were identified among males, black, unmarried, smokers, with no religious affiliation, and persons without chronic disease attended at the health unit. Health services should therefore be prepared to identify the early stages of problematic alcohol abuse in order to prevent social and health consequences in the population, especially those attended in primary health care, for which the promotion of health and prevention of disease are priority health concerns.

Key words Alcohol drinking, Prevention and control, Primary health care, Epidemiological surveys, Brazil
Resumo Objetivou-se estimar os padrões de consumo de álcool e identificar fatores associados entre usuários adultos de um serviço de atenção básica no município do Rio de Janeiro, Brasil. Desenvolvido através de inquérito domiciliar com amostra de 301 indivíduos que responderam a um instrumento de coleta que continha o Alcohol Use Disorders Identification Test e variáveis de caracterização sociodemográfica e de saúde. A regressão logística foi utilizada para verificar associação entre o padrão de consumo problemático de álcool e as variáveis independentes. Identificaram-se padrões de consumo problemático de álcool entre pessoas do sexo masculino, de cor da pele negra elou mestiça, não casadas, sem religião, tabagistas e entre pessoas sem doença crônica acompanhada pela unidade de saúde. Os serviços de saúde devem, portanto, estar preparados para identificar precocemente pessoas que fazem consumo problemático de álcool, a fim de prevenir consequências sociais e de saúde nas populações, especialmente nas atendidas pela atenção básica, que tem a promoção da saúde e a prevenção de agravos como práticas sanitárias prioritárias.

Palavras-chave Consumo de bebidas alcoólicas, Prevenção e controle, Atenção Primária à Saúde, Inquéritos Epidemiológicos, Brasil 


\section{Introdução}

O álcool é uma droga psicotrópica que tem o consumo admitido e até mesmo incentivado pela sociedade, apesar de seu consumo abusivo ser importante problema de saúde pública, pois tem sido apontado como responsável por grande número de acidentes de trânsito e de trabalho, violência doméstica e aumento da morbi-mortalidade por doenças cardiovasculares, cirrose hepática, acidentes vasculares cerebrais e distúrbios psiquiátricos ${ }^{1}$. O consumo de álcool é considerado ainda o primeiro fator de risco para a carga global de doenças em países das Américas e seu consumo abusivo está relacionado à cerca de 2,5 milhões de mortes anuais em todo o mundo ${ }^{2}$.

Em relação ao Brasil, a comparação dos resultados de dois levantamentos domiciliares sobre o uso de drogas realizados nos anos de $2001 \mathrm{e}$ 2005 identificou aumento significativo de uso de álcool na vida pelos brasileiros ${ }^{3}$. A comparação dos resultados dos anos de 2006 a 2009 do sistema contínuo de monitoramento de fatores de risco e proteção para doenças crônicas por inquérito telefônico na população brasileira (VIGITEL) apontou uma tendência crescente do consumo abusivo de álcool em ambos os sexos ${ }^{4}$. Estudo feito com amostra representativa da população brasileira adulta de todo o território nacional, entre os anos de 2005 e 2006, aponta que $9 \%$ dela preenche critérios de dependência alcoólica ${ }^{5}$.

Vale ressaltar, no entanto, que o conceito de consumo problemático de álcool não se aplica somente ao consumidor abusivo ou ao dependente, pois há outros padrões de consumo que podem causar riscos substanciais ou nocivos para o indivíduo. Entre eles, a situação de beber muito todos os dias, repetidos episódios de intoxicação, beber de forma que cause prejuízo físico ou mental $\mathrm{e} o$ ato de beber que resulte na pessoa se tornar dependente. O consumo problemático de álcool, portanto, pode se estender em um processo contínuo, desde um padrão de beber excessivo até a dependência, ou seja, em um continuum ${ }^{6}$.

Em âmbito populacional, este continuum deve ser levado em consideração, pois não existe um limiar de exposição ao álcool abaixo do qual o risco de adoecer seja nulo, o que faz com que ações de promoção e de vigilância à saúde sejam fundamentais para prevenir a ocorrência de enfermidades e agravos relacionados ao uso dessa substância, especialmente as desenvolvidas na atenção básica $(\mathrm{AB})$, espaço que privilegia tais ações e principal porta de entrada dos usuários no sistema de saúde brasileiro.
Portanto, a realização de rastreamentos para identificação dos padrões de consumo de álcool entre as populações é importante pelo fato de a maioria das pessoas que faz uso abusivo de álcool não ser diagnosticada até que tenham desenvolvido sérias complicações decorrentes de tal uso $^{6,7}$. Ademais, a maioria dos usuários dos serviços de $\mathrm{AB}$ que consome álcool em níveis de risco não apresenta necessariamente quadro de dependência à substância, pois a maior parte dos problemas ocasionados pelo seu consumo manifesta-se entre aqueles que não são dependentes, mas que apresentam comportamento de risco quando bebem ${ }^{6,8}$.

Em virtude da grande prevalência e dos problemas relacionados ao consumo de álcool no Brasil e considerando a importância dos serviços de $\mathrm{AB}$, estratégicos para a implementação de ações de identificação precoce e de prevenção de agravos à saúde, é oportuna a realização de estudos que caracterizem o consumo de álcool entre usuários desses serviços ${ }^{6-11}$. Portanto, o objetivo deste estudo foi estimar os padrões de consumo de álcool e identificar fatores associados entre usuários adultos de um serviço de $\mathrm{AB}$ no município do Rio de Janeiro, Brasil.

\section{Métodos}

Inquérito domiciliar desenvolvido em uma unidade básica de saúde (UBS) que funciona no modelo da Estratégia Saúde da Família situada na zona oeste do município do Rio de Janeiro, Brasil. A UBS em questão era responsável pelos cuidados básicos de saúde de 587 famílias, que totalizavam 3.476 pessoas.

Os participantes deste estudo foram selecionados por um processo de amostragem aleatória simples feito no software Epi-Info versão 3.5.1 para cálculo amostral em inquéritos, a partir da seguinte fórmula:

$\mathrm{n}=\mathrm{Nz}^{2} \mathrm{p}(1-\mathrm{p}) /\left[\mathrm{d}^{2}(\mathrm{~N}-1)+\mathrm{z}^{2} \mathrm{p}(1-\mathrm{p})\right]^{\mathrm{a}}$

Onde $\mathrm{N}$ = população total de adultos na faixa etária considerada; $\mathrm{z}=$ valor correspondente ao nível de confiança ao quadrado $\left(1,96^{2}=3,84\right)$; $\mathrm{d}=$ precisão absoluta ao quadrado $\left(0,06^{2}=\right.$ $0,0036) ; \mathrm{p}=$ proporção da população com a característica em estudo $(0,5)$.

O tamanho da amostra calculado foi de 279 pessoas, no entanto, planejou-se entrevistar 307 , estimando-se uma perda em torno de $10 \%$ por recusa ou outros motivos. Para a seleção dos 307 indivíduos, seguiram-se as seguintes etapas: todas as 3.476 fichas cadastrais da UBS foram ana- 
lisadas, sendo separadas e numeradas aquelas pertencentes aos 1.016 adultos cadastrados que atendiam aos critérios de inclusão (idade entre 20-59 anos) e exclusão (indivíduos com incapacidade mental/cognitiva e gestantes) do estudo. Em seguida, foram sorteados os 307 indivíduos, a partir das fichas cadastrais numeradas previamente.

As visitas aos domicílios não foram agendadas e aconteceram nos meses de novembro e dezembro de 2010: das 09h às 20h, de segundafeira à sexta-feira, e, das 09 às $12 \mathrm{~h}$, aos sábados, domingos e feriados. Os indivíduos foram entrevistados por único pesquisador em locais reservados e sem a presença de terceiros, através de um instrumento de coleta de dados que continha o questionário Alcohol Use Disorders Identification Test (AUDIT) e variáveis de caracterização sociodemográfica (sexo, faixa etária, cor da pele, escolaridade, situação conjugal, situação trabalhista, renda per capita e religião) e de saúde (tabagismo, autoavaliação de saúde e acompanhamento de doença crônica na UBS).

A seção do instrumento de coleta de dados que se relacionava à caracterização sociodemográfica e de saúde foi aprimorada através de 11 rodadas de pré-testes, quanto ao que se refere à ordem das perguntas e clareza no entendimento dos entrevistados, em amostra de voluntários que não fizeram parte da amostra final do estudo. Com o objetivo de testar a logística do estudo, foi realizado um estudo-piloto com 20 usuários da UBS que também não fizeram parte da amostra final deste estudo.

O AUDIT, desenvolvido pela Organização Mundial de Saúde ${ }^{6}$ e já validado no Brasil ${ }^{12}$, classifica o padrão de consumo de álcool com base no nível de risco. Para cada resposta dada pelo indivíduo a uma de suas dez questões há um escore que pode variar de 0 a 4 pontos. Ao final da aplicação do AUDIT, os escores apresentados em cada questão são somados e, a partir disto, o padrão de consumo de álcool do indivíduo é classificado. Portanto, os participantes desta pesquisa foram classificados em uma das quatro possíveis zonas de risco de consumo de álcool, a saber:

Zona de Risco I - indivíduos que tinham um padrão de consumo de baixo risco de álcool ou eram abstêmios. Pontuação entre 0 e 7.

Zona de Risco II - indivíduos que tinham um padrão de consumo de risco de álcool. Pontuação entre 8 e 15.

Zona de Risco III - indivíduos que tinham um padrão de consumo nocivo de álcool. Pontuação entre 16 e 19.
Zona de Risco IV - indivíduos que tinham um padrão de consumo de provável dependência de álcool. Pontuação entre 20 e 40.

$\mathrm{Na}$ fase analítica dos dados, a revisão e a codificação das questões foram realizadas e, em seguida, as informações contidas nos instrumentos de coleta dos dados foram digitadas no software Epi-Info versão 3.5.1. No software SPSS versão 13.0 foram realizadas as análises univariadas com distribuição de frequências simples para a descrição da população e também as análises bivariadas, verificando a associação entre cada variável independente do estudo e os padrões de consumo de álcool (desfecho). Estas análises foram baseadas no teste do qui-quadrado de Pearson, adotando-se nível de significância de 0,05.

Os resultados dos testes de qui-quadrado de Pearson durante as análises bivariadas foram observados e a regressão logística foi utilizada como procedimento de análise multivariada para controlar as possíveis variáveis de confusão naquelas que apresentaram p-valor $<0,10$. Na análise multivariada a variável dependente foi classificada de forma dicotômica, utilizando-se como ponto de corte o escore 8 do AUDIT ${ }^{13}$ (padrão de consumo problemático de álcool) e considerando-se os escores de 0-7 como categoria de referência.

\section{Aspectos Éticos}

Este estudo foi aprovado pelo Comitê de Ética em Pesquisa da Secretaria Municipal de Saúde e Defesa Civil do Rio de Janeiro. Conforme acordado previamente com a Gerência Técnica da UBS, os indivíduos que foram identificados como prováveis dependentes de álcool receberam encaminhamento por escrito para a unidade básica, a fim de que a equipe o acompanhasse e providenciasse novo encaminhamento para a um serviço de referência especializada.

\section{Resultados}

Dos 307 indivíduos selecionados, houve perda de $6(1,95 \%)$ por recusas na participação do estudo, compondo a amostra final, então, 301 sujeitos com idade compreendida entre 20 e 59 anos (média de idade de 39,2 $\pm 12,0$ anos), sendo pouco mais da metade $(59,8 \%)$ mulheres. Em relação à cor da pele, $51,1 \%$ dos indivíduos se autodeclararam mestiços. Em relação à escolaridade, metade dos entrevistados (50,5\%) informou possuir até o ensino fundamental completo e a maioria 
$(65,4 \%)$ era casada ou vivia em união estável. No tocante à religião, 38,2\% dos sujeitos informaram ser evangélicos. No que concerne à situação trabalhista dos indivíduos, pouco mais da metade $(65,8 \%)$ estava empregada. A renda per capita média foi de $\mathrm{R} \$ 418,72 \pm \mathrm{R} \$ 451,77$, sendo que $42,9 \%$ dos entrevistados possuía renda per capta de até $1 / 2$ salário mínimo $(\mathrm{R} \$ 255,00)$ e somente $20,2 \%$ possuía renda per capta igual ou superior a 1 salário mínimo ( $\mathrm{R} \$ 510,00)$ (Tabela 1).

Observou-se que $12,3 \%$ dos sujeitos declararam-se fumantes e $68,1 \%$ nunca terem fumado. Quanto à autopercepção de saúde, 55,7\% informaram considerá-la boa e 4,3\% ruim. Afirmaram ser acompanhados pela USF por alguma doença crônica 27,6\% dos entrevistados. Quanto aos padrões de consumo de álcool, 70,4\% dos sujeitos faziam consumo de baixo risco e 17,6\% faziam consumo de risco de álcool (Tabela 1). Ressalta-se que metade dos entrevistados (50,5\%) afirmou nunca consumir bebida alcoólica.

Entre indivíduos do sexo masculino identificaram-se proporções mais elevadas de consumo de risco, nocivo e provável dependência de álcool quando comparadas às proporções dos mesmos padrões de consumo das mulheres $(\mathrm{p}=0,006)$. Entre os não casados, as proporções de consumo de risco, nocivo e provável dependência de álcool foram maiores que as dos casados ( $\mathrm{p}<$ $0,001)$. Indivíduos empregados tiveram proporções maiores de consumo de risco, nocivo e provável dependência de bebidas alcoólicas quando comparadas às dos não empregados $(\mathrm{p}=0,005)$. Indivíduos que possuíam religião apresentaram maior proporção de consumo de baixo risco $(82,3 \%)$ quando comparada a proporção do mesmo tipo de consumo de indivíduos sem religião (50,0\%). Assim, as proporções de consumo de risco, nocivo e provável dependência de álcool dos indivíduos que não possuíam religião foram maiores que as dos indivíduos que a possuíam $(\mathrm{p}<0,001)$ (Tabela 2).

As proporções de consumo de risco, nocivo e provável dependência de álcool foram maiores entre os indivíduos fumantes quando comparadas às proporções dos mesmos padrões de consumo de pessoas que nunca fumaram. A proporção de ex-fumantes e de nunca fumantes que faziam consumo de baixo risco de álcool foi cerca de mais de duas vezes mais elevada que do que aquela observada entre os atuais fumantes ( $\mathrm{p}<$ $0,001)$. Também foram identificadas proporções maiores dos consumos de risco, nocivo e provável dependência de álcool em indivíduos que avaliaram a própria saúde regular ou ruim quando comparadas às proporções dos mesmos padrões de consumo dos sujeitos que a avaliaram como muito boa ou boa $(\mathrm{p}=0,004)$. Portadores de doença crônica acompanhada pela UBS tiveram menores proporções de consumo de risco, nocivo e provável dependência do álcool quando comparadas às proporções de indivíduos que não eram portadores de doença crônica $(\mathrm{p}=0,004)$ (Tabela 2).

No modelo de regressão logística multivariada observaram-se maiores chances de consumo problemático de alcool entre os homens, embora a associação tenha se mostrado limítrofe (OR $=1,77$; IC $95 \%=0,94-3,31$ ), entre os não casados $(\mathrm{OR}=2,97)$ e entre aqueles que referiram não possuir religião $(\mathrm{OR}=3,37)$. Além disso, as chances foram mais elevadas entre aqueles que se autoreferiram mestiços e negros quando comparados aos brancos $(\mathrm{OR}=2,95$ e $\mathrm{OR}=4,83)$. Quando comparados aos entrevistados que referiram não fumar os fumantes atuais apresentaram chances cinco vezes mais elevadas de consumo problemático do álcool $(\mathrm{OR}=5,65)$, entre ex-fumantes a direção da associação foi para a proteção, embora sem significância estatística. Ademais, a chance do consumo problemático do álcool foi cerca de três vezes mais elevada entre aqueles que referiram não fazer acompanhamento para doença crônica no serviço de saúde da área $(\mathrm{OR}=3,27)$ (Tabela 3$)$.

\section{Discussão}

No presente estudo foram identificados padrões de consumo problemático de álcool principalmente entre pessoas do sexo masculino, de cor da pele negra e/ou mestiça, não casadas, sem religião, tabagistas e entre pessoas sem doença crônica acompanhada pela UBS. Vale ressaltar que a identificação do perfil de consumo de álcool é útil para o entendimento da distribuição dos aspectos ligados a este comportamento e capaz de oferecer uma melhor compreensão a respeito das características sociodemográficas ligadas a grupos com maior exposição a fatores e/ou comportamentos de risco, contribuindo para a elaboração de políticas e programas de saúde mais eficazes e equânimes ${ }^{14}$.

A proporção de abstêmios identificados na população estudada foi semelhante à de outros estudos populacionais ${ }^{5,15-17}$, bem como os padrões de consumo de álcool, ${ }^{5,10,17}$. Apesar de o consumo de bebidas alcoólicas ser socialmente aceito, o grau de abstinência da população estu- 
Tabela 1. Características sociodemográficas, características relacionadas à saúde e padrões de consumo de álcool da amostra estudada. Rio de Janeiro, RJ, Brasil, 2010. ( $\mathrm{n}=301)$

\begin{tabular}{|c|c|c|}
\hline Variáveis & $\mathbf{n}$ & $\%$ \\
\hline \multicolumn{3}{|l|}{ Sexo } \\
\hline Feminino & 180 & 59,8 \\
\hline Masculino & 121 & 40,2 \\
\hline \multicolumn{3}{|l|}{ Faixa etária } \\
\hline Média (DP) & $39,2(12,0)$ & Variação: 20 a 59 anos \\
\hline 20-39 & 157 & 52,2 \\
\hline 40-59 & 144 & 47,8 \\
\hline \multicolumn{3}{|l|}{ Cor da pele autorreferida } \\
\hline Branca & 64 & 21,3 \\
\hline Negra & 83 & 27,6 \\
\hline Mestiça & 154 & 51,1 \\
\hline \multicolumn{3}{|l|}{ Escolaridade } \\
\hline Nenhuma & 10 & 3,3 \\
\hline Até o ensino fundamental completo & 152 & 50,5 \\
\hline$\geq$ Ensino médio incompleto & 139 & 46,2 \\
\hline \multicolumn{3}{|l|}{ Situação conjugal } \\
\hline Casado/Vive em união & 197 & 65,4 \\
\hline Divorciado/Separado & 51 & 16,9 \\
\hline Viúvo & 21 & 7,0 \\
\hline Solteiro & 32 & 10,6 \\
\hline \multicolumn{3}{|l|}{ Situação trabalhista } \\
\hline Empregado & 198 & 65,8 \\
\hline Desempregado & 60 & 19,9 \\
\hline Aposentado/Pensionista & 36 & 12,0 \\
\hline Não se aplica & 07 & 2,3 \\
\hline \multicolumn{3}{|l|}{ Renda per capita } \\
\hline Média (DP) & $418,72(451,77)$ & Variação: 0 a 4500 \\
\hline $\mathrm{R} \$ 0$ a 255 (até $1 / 2 \mathrm{SM})$ & 129 & 42,9 \\
\hline $\mathrm{R} \$ 255$ a $510(1 / 2$ a $1 \mathrm{SM})$ & 111 & 36,9 \\
\hline$\geq \mathrm{R} \$ 510(\geq 1 \mathrm{SM})$ & 61 & 20,2 \\
\hline \multicolumn{3}{|l|}{ Religião } \\
\hline Não possui & 108 & 35,8 \\
\hline Católica & 71 & 23,5 \\
\hline Evangélica & 115 & 38,4 \\
\hline Outras & 07 & 2,3 \\
\hline \multicolumn{3}{|l|}{ Tabagismo } \\
\hline Fumante & 37 & 12,3 \\
\hline Ex-fumante & 59 & 19,6 \\
\hline Nunca fumou & 205 & 68,1 \\
\hline \multicolumn{3}{|l|}{ Autoavaliação de saúde } \\
\hline Muito boa & 43 & 14,3 \\
\hline Boa & 167 & 55,7 \\
\hline Regular & 77 & 25,7 \\
\hline Ruim & 13 & 4,3 \\
\hline \multicolumn{3}{|l|}{ Doença crônica acompanhada pela UBS } \\
\hline $\operatorname{Sim}$ & 83 & 27,6 \\
\hline Não & 218 & 72,4 \\
\hline \multicolumn{3}{|l|}{ Padrão de consumo de álcool } \\
\hline Consumo de baixo risco & 212 & 70,4 \\
\hline Consumo de risco & 53 & 17,6 \\
\hline Consumo nocivo & 19 & 6,3 \\
\hline Provável dependência & 17 & 5,7 \\
\hline
\end{tabular}


Tabela 2. Associação das características sociodemográficas e características relacionadas à saúde com os padrões de consumo de álcool da amostra estudada. Rio de Janeiro, RJ, Brasil, 2010. ( $\mathrm{n}=301)$

\begin{tabular}{|c|c|c|c|c|c|c|c|c|c|}
\hline & \multicolumn{9}{|c|}{ Padrões de consumo de álcool } \\
\hline & \multicolumn{2}{|c|}{$\begin{array}{c}\text { Baixo } \\
\text { risco }\end{array}$} & \multicolumn{2}{|c|}{ Risco } & \multicolumn{2}{|c|}{ Nocivo } & \multicolumn{2}{|c|}{$\begin{array}{c}\text { Provável } \\
\text { dependência }\end{array}$} & \multirow{2}{*}{ p-valor } \\
\hline & $\mathbf{n}$ & $\%$ & $\mathbf{n}$ & $\%$ & $\mathbf{n}$ & $\%$ & $\mathbf{n}$ & $\%$ & \\
\hline \multicolumn{10}{|l|}{ Sexo } \\
\hline Masculino & 73 & 60,3 & 25 & 20,7 & 12 & 9,9 & 11 & 9,1 & \\
\hline Feminino & 139 & 77,2 & 28 & 15,6 & 07 & 3,9 & 06 & 3,3 & 0,006 \\
\hline \multicolumn{10}{|l|}{ Faixa etária } \\
\hline 20-39 & 108 & 68,8 & 26 & 16,6 & 13 & 8,3 & 10 & 6,4 & \\
\hline $40-59$ & 104 & 72,2 & 27 & 18,8 & 06 & 4,2 & 07 & 4,9 & 0,449 \\
\hline \multicolumn{10}{|l|}{ Cor da pele autorreferida } \\
\hline Branca & 52 & 81,3 & 7 & 10,9 & 04 & 6,3 & 01 & 1,6 & \\
\hline Negra & 49 & 59,0 & 16 & 19,3 & 09 & 10,8 & 09 & 10,8 & 0,080 \\
\hline Mestiça & 111 & 71,9 & 30 & 19,6 & 06 & 3,9 & 07 & 4,6 & \\
\hline \multicolumn{10}{|l|}{ Escolaridade } \\
\hline Até fundamental completo & 110 & 67,9 & 33 & 20,4 & 08 & 4,9 & 11 & 6,8 & 0,296 \\
\hline$\geq$ Médio incompleto & 102 & 73,4 & 20 & 14,4 & 11 & 7,9 & 06 & 4,3 & \\
\hline \multicolumn{10}{|l|}{ Situação conjugal } \\
\hline Casados & 154 & 78,2 & 31 & 15,7 & 06 & 3,0 & 06 & 3,0 & \\
\hline Não casados & 58 & 55,8 & 22 & 21,2 & 13 & 12,5 & 11 & 10,6 & $<0,001$ \\
\hline \multicolumn{10}{|l|}{ Situação trabalhista } \\
\hline Empregados & 131 & 66,2 & 36 & 18,2 & 16 & 8,1 & 15 & 7,6 & \\
\hline Não empregados & 81 & 78,6 & 17 & 16,5 & 03 & 2,9 & 02 & 1,9 & 0,005 \\
\hline \multicolumn{10}{|l|}{ Renda per capita } \\
\hline $\mathrm{R} \$ 0$ a 255 & 86 & 66,7 & 23 & 17,8 & 10 & 7,8 & 10 & 7,8 & \\
\hline $\mathrm{R} \$ 255$ a 510 & 81 & 73,0 & 20 & 18,0 & 06 & 5,4 & 04 & 3,6 & 0,704 \\
\hline$\geq \mathrm{R} \$ 510$ & 45 & 75 & 10 & 16,7 & 03 & 5,0 & 02 & 3,3 & \\
\hline \multicolumn{10}{|l|}{ Religião } \\
\hline Possui & 159 & 82,3 & 26 & 13,5 & 03 & 1,6 & 05 & 2,6 & \\
\hline Não possui & 54 & 50,0 & 27 & 25,9 & 16 & 14,8 & 11 & 10,2 & $<0,001$ \\
\hline \multicolumn{10}{|l|}{ Tabagismo } \\
\hline Fumante & 13 & 35,1 & 12 & 32,4 & 08 & 21,6 & 04 & 10,8 & \\
\hline Ex-fumante & 47 & 79,9 & 06 & 10,2 & 0 & 0,0 & 06 & 10,2 & \\
\hline Nunca fumou & 151 & 72,4 & 34 & 16,7 & 11 & 5,4 & 07 & 3,4 & $<0,001$ \\
\hline \multicolumn{10}{|l|}{ Auto-avaliação de saúde } \\
\hline Muito boa/Boa & 155 & 73,8 & 36 & 17,1 & 10 & 4,8 & 09 & 4,3 & \\
\hline Regular/Ruim & 56 & 62,2 & 17 & 18,9 & 09 & 10,0 & 08 & 8,9 & 0,015 \\
\hline \multicolumn{10}{|l|}{$\begin{array}{l}\text { Acompanhamento de doença } \\
\text { crônica pela UBS }\end{array}$} \\
\hline Sim & 70 & 84,3 & 09 & 10,8 & 01 & 1,2 & 03 & 3,6 & \\
\hline Não & 142 & 65,1 & 44 & 20,2 & 18 & 8,3 & 14 & 6,4 & 0,004 \\
\hline
\end{tabular}

dada foi alto. Em contrapartida, os bebedores apresentaram elevado nível de consumo problemático de álcool, o que aponta para a importância dos profissionais da UBS em questão e também para as políticas públicas brasileiras considerarem o desenvolvimento de ações de prevenção ao consumo problemático de álcool.

Neste estudo a participação de mulheres foi um pouco maior que a dos homens, sendo que, entre elas, identificaram-se menores padrões de consumo problemático de álcool. Além disso, quando foram analisados os padrões de consumo de risco, nocivo e de provável dependência entre os sexos, foram os homens que se destacaram, apresentando proporções superiores às das mulheres. Tais resultados corroboram com o que tem sido amplamente descrito na literatu$\mathrm{ra}^{5,7,10,15,16,18-21}$. 
Tabela 3. Associações brutas e ajustadas de variáveis selecionadas e o consumo problemático de álcool. Rio de Janeiro, RJ, Brasil, 2010

\begin{tabular}{|c|c|c|}
\hline & OR bruta (IC 95\%) & OR ajustada (IC 95\%) \\
\hline \multicolumn{3}{|l|}{ Sexo } \\
\hline Feminino & 1,0 & 1,0 \\
\hline Masculino & $2,08(1,25-3,45)$ & $1,77(0,94-3,31)^{* *}$ \\
\hline \multicolumn{3}{|l|}{ Faixa etária } \\
\hline 20-39 & 1,0 & 1,0 \\
\hline $40-59$ & $0,79(0,48-1,30)$ & $1,64(0,77-3,50)$ \\
\hline \multicolumn{3}{|l|}{ Cor da pele } \\
\hline Brancos & 1,0 & 1,0 \\
\hline Mestiça & $1,82(0,87-3,82)$ & $2,95(1,58-5,58)$ \\
\hline Negra & $3,34(1,53-7,31)$ & $4,83(1,79-13,01)$ \\
\hline \multicolumn{3}{|l|}{ Situação conjugal } \\
\hline Casados & 1,0 & 1,0 \\
\hline Não casados & $3,02(1,79-5,06)$ & $2,97(1,58-5,58)$ \\
\hline \multicolumn{3}{|l|}{ Situação Trabalhista } \\
\hline Empregados & 1,0 & 1,0 \\
\hline Não empregados & $0,55(0,32-0,97)$ & $0,55(0,27-1,12)$ \\
\hline \multicolumn{3}{|l|}{ Religião } \\
\hline Possui & 1,0 & 1,0 \\
\hline Não possui & $5,0(2,93-8,54)$ & $3,37(1,77-6,39)$ \\
\hline \multicolumn{3}{|l|}{ Tabagismo } \\
\hline Nunca fumou & 1 & 1 \\
\hline Ex-fumante & $0,72(0,31-1,70)$ & $0,50(0,38-1,59)$ \\
\hline Fumante & $4,19(1,59-11,04)$ & $5,65(2,67-11,92)$ \\
\hline \multicolumn{3}{|c|}{ Auto-avaliação de saúde } \\
\hline Muito boa/Boa & 1 & 1 \\
\hline Ruim/Regular & $1,79(1,06-3,05)$ & $1,59(0,79-3,16)$ \\
\hline \multicolumn{3}{|c|}{ Acompanhamento de doença crônica } \\
\hline Sim & 1 & 1 \\
\hline Não & $3,10(1,59-6,08)$ & $3,27(1,31-8,15)$ \\
\hline
\end{tabular}

"Ajustada para todas as variáveis da tabela. ${ }^{* *}$ IC limítrofe.

No que concerne à associação entre as faixas etárias estudadas e os padrões de consumo de álcool, não houve diferença estatisticamente significante entre elas, apesar de outros estudos terem encontrado associação inversa entre idade e consumo problemático de álcool ${ }^{5,20,21}$. Isto pode ser parcialmente explicado pela inclusão de sujeitos nos outros estudos com idade inferior a $20 \mathrm{e}$ superior a 60 anos, e neste ter se limitado de 20 a 59 anos, tornando sua população mais homogênea no que se refere às faixas etárias e seus resultados pouco comparáveis.

Segundo o I Levantamento Nacional sobre os Padrões de Consumo de Álcool na População Brasileira $^{22}$ a frequência do beber varia muito no que diz respeito às faixas etárias: enquanto o índice de beber diário é relativamente constante entre as faixas etárias, a abstinência é mais de
$79 \%$ maior entre os brasileiros de 60 anos ou mais do que entre os jovens de 18 a 24 anos.

Foi encontrado baixo nível de escolaridade entre os entrevistados, pois mais da metade deles possuíam apenas o ensino fundamental completo ou incompleto. A proporção de sujeitos prováveis dependentes, entre aqueles que possuíam até o ensino fundamental completo, foi maior que a encontrada entre os que possuíam o ensino médio completo ou mais. Assim como neste estudo, a literatura tem demonstrado tendência de maiores taxas de dependência alcoólica em grupos sociais com baixa escolaridade ${ }^{21,23,24}$.

Vale ressaltar que a oportunidade de educação formal é um fator determinante sobre as condições de saúde dos indivíduos ${ }^{25}$, ademais, sabe-se que existem iniquidades na utilização de serviços preventivos de saúde em relação a pes- 
soas de baixa escolaridade ${ }^{26}$. Como a proposta da $A B$ busca ressaltar uma concepção de assistência à saúde, através de ações preventivas, intervenções sobre os fatores de risco e do desenvolvimento da qualidade de vida, as ações preventivas em saúde, especialmente às de controle do consumo excessivo de álcool, devem estar voltadas para grupos mais vulneráveis.

Indivíduos negros e/ou mestiços apresentaram níveis mais elevados de consumo problemático de álcool neste estudo. Em pesquisa realizada em 2005 no Brasil com 5.040 indivíduos de ambos os sexos de todas as regiões do país, ser da cor branca mostrou-se como fator de proteção para o consumo regular de álcool ${ }^{27}$.

Acredita-se que os resultados do presente estudo sejam reflexos das iniquidades raciais em saúde, expressas pelos diferenciais nos riscos de adoecer e de morrer, originados de condições heterogêneas de existência e de acesso a bens e serviços. As desigualdades nos indicadores de saúde entre a variável raça/cor de pele remetem, portanto, aos determinantes sociais de saúde como um fator influente na pior condição de saúde para negros comparados aos brancos ${ }^{28}$, no caso do presente estudo, o consumo problemático de álcool.

Neste contexto, destaca-se a Política Nacional de Saúde Integral da População Negra ${ }^{29}$ que tem o propósito de garantir maior grau de equidade no que tange à efetivação do direito humano à saúde, em seus aspectos de promoção, prevenção, atenção, tratamento e recuperação de doenças e agravos, incluindo aqueles de maior prevalência nesse segmento populacional. Logo, a equipe da UBS onde o estudo foi desenvolvido deve considerar estes resultados e dirigir ações preventivas relacionadas ao consumo problemático de álcool especialmente aos negros usuários de seus serviços.

A situação conjugal também se mostrou associada aos padrões problemáticos de consumo de álcool no presente estudo. Entre os não casados observaram-se padrões de consumo de álcool de maior risco do que entre os casados. Resultados semelhantes foram encontrados em outros estudos ${ }^{18,30-32}$.

Estudos apontam que indivíduos solteiros apresentam maior chance de manifestar e manter o uso abusivo de álcool ${ }^{33}$ e estar casado ou ter companheiro seja fator protetor para o consumo problemático de álcool ${ }^{34}$. Nesse sentido, a união estável parece funcionar como um fator de proteção para o consumo de álcool, especialmente entre os homens, já que as mulheres costumam con- tribuir para que os mesmos tenham mais cuidados com a própria saúde, aconselhando-os, inclusive, a não consumir álcool excessivamente.

No tocante à situação trabalhista dos sujeitos deste estudo, na análise multivariada a variável empregado perdeu significância estatística. Entretanto, outros estudos encontraram associação entre situação trabalhista (empregado) e consumo abusivo de álcool ${ }^{16,17,19}$. Ademais, não estar empregado mostrou-se fator de proteção para consumo problemático de álcool, o que pode ser parcialmente explicado pelos aposentados e donas de casa entrevistados se enquadrarem nesta "situação trabalhista", e sabe-se que idosos (grupo de indivíduos em que a maioria é aposentada) e mulheres, quando consomem álcool, tendem a consumi-lo em um padrão de baixo risco ${ }^{22}$. Vale destacar, no entanto, que a relação entre consumo de álcool e situação trabalhista tem sido pouco estudada e necessita de maior investigação no que diz respeito ao maior uso na população economicamente ativa ${ }^{19}$.

Neste estudo, indivíduos que não possuíam religião apresentaram maiores chances de padrão de consumo problemático quando comparados àqueles que possuíam. Resultados semelhantes também foram encontrados em outros estu$\operatorname{dos}^{10,17,27,35}$. Segundo Bastos et al. ${ }^{27}$, o fato de o indivíduo não ser criado em lar onde a religião se mostra relevante também pode aumentar a chance de adoção do consumo abusivo de álcool. Nesse sentido, a prática religiosa parece funcionar como fator de proteção para o consumo problemático dessa substância ${ }^{35,36}$. Tal ocorrência pode estar relacionada ao fato de algumas religiões, especialmente as de origem cristã, pregarem a abstinência alcoólica.

Os padrões de consumo de risco, nocivo e provável dependência de álcool foram muito elevados entre indivíduos que se autodeclararam fumantes em comparação aos não fumantes. Resultados semelhantes também foram encontrados em outros estudos nacionais ${ }^{20,21,37} \mathrm{e}$ internacionais ${ }^{38,39}$. O consumo de álcool e o tabagismo são dois fatores de risco modificáveis para doenças crônicas não transmissíveis e o uso combinado aumenta o risco de câncer da cavidade bucal e de esôfago e também em outras localizações, como a faringe e a laringe supraglótica. Estima-se que das pessoas com câncer na cavidade bucal $76 \%$ delas fumem e consumam bebidas alcoólicas regularmente ${ }^{40}$.

Mesmo não apresentando significância estatística na análise multivariada, os padrões de consumo problemático de álcool entre os sujeitos que 
avaliaram sua saúde como regular ou ruim foram superiores aos daqueles que a avaliaram como boa ou muito boa. Resultados semelhantes foram encontrados em outros estudos ${ }^{19,41}$. Diante disso, levar em consideração a autoavaliação de saúde pode ser útil para que os profissionais de UBS abordem questões relacionadas aos maus hábitos de saúde, inclusive o consumo excessivo de álcool, promovendo a adoção de comportamentos saudáveis, colaborando, assim, com a minimização dos agravos decorrentes deles.

Associação entre acompanhamento de doença crônica na UBS e o padrão de consumo de baixo risco de álcool foi encontrada neste estudo, bem como associação entre não ser doente crônico e padrões de uso problemático. É possível que os portadores de doenças crônicas que participaram deste estudo adotassem um padrão de consumo de baixo risco de álcool devido ao acompanhamento regular de sua condição pela equipe de Saúde da Família, que certamente promove a adoção de comportamentos saudáveis entre eles, tais como a redução da gordura e do sal na dieta, a promoção de atividade física, bem como a redução ou cessação do consumo de bebidas alcoólicas, entre outros.

As doenças crônicas não transmissíveis são responsáveis por $61 \%$ de todas as mortes ocorridas no mundo e um pequeno conjunto de fatores de risco responde pela grande maioria delas. Dentre esses fatores de risco, além do consumo excessivo de bebidas alcoólicas, encontram-se o tabagismo, a obesidade, as dislipidemias, a ingestão insuficiente de frutas, legumes e verduras e a inatividade física $^{42}$. O Brasil está entre os países com altas taxas de morbimortalidade atribuíveis ao consumo de álcool ${ }^{43}$, estando algumas doenças crônicas associadas ao seu consumo de risco e nocivo, porém o desenvolvimento dessas doenças pode se iniciar mesmo com a ingestão de quantidades não consideradas de risco ${ }^{44}$.

À luz dos resultados encontrados no presente estudo, ressalta-se que os serviços de saúde devem estar preparados para identificar precocemente pessoas que fazem consumo problemático de álcool. Em especial, os serviços de AB que devem voltar sua atenção também para práticas preventivas, educativas e terapêuticas relacionadas ao consumo abusivo dessa substância.

No entanto, são conhecidas as dificuldades dos serviços e dos profissionais de saúde para diagnosticar, motivar, tratar ou encaminhar para tratamento os pacientes que fazem consumo problemático de álcool, especialmente quando a atenção desses profissionais está direcionada para a dependência alcoólica ${ }^{45}$, apesar de parte substancial dos problemas clínicos e de saúde pública ocorrer nos chamados bebedores de risco ${ }^{46}$. Portanto, avaliar sistematicamente os padrões de consumo de álcool é fundamental para prevenir consequências sociais e de saúde nas populações, especialmente naquelas atendidas pela $\mathrm{AB}$ que tem a promoção/proteção da saúde e a prevenção de agravos como práticas sanitárias prioritárias.

Nesse sentido, é essencial a aproximação dos profissionais das necessidades de saúde da população atendida pela $\mathrm{AB}$ levando em consideração a relevância do consumo abusivo de álcool, por isso a identificação precoce de tal consumo já neste nível de atenção poderá auxiliar no desenvolvimento de estratégias para a prevenção de problemas por ele causados. Então, é importante priorizar as ações de promoção de saúde, com ênfase na redução do consumo de álcool, entre os grupos mais vulneráveis ao seu padrão de consumo problemático de forma a evitar a manutenção de tal padrão bem como sua evolução para casos de dependência.

Diante disso, vale destacar que a aplicação rotineira do AUDIT durante as consultas médicas e de enfermagem realizadas na $\mathrm{AB}$, especialmente nos serviços vinculados à Estratégia Saúde da Família, pode facilitar a abordagem dos usuários desses serviços em relação ao uso de bebidas alcoólicas, auxiliando, assim, na condução de intervenções e práticas preventivas individualizadas e centradas na singularidade da pessoa identificada como usuária de risco ${ }^{47}$.

Convém mencionar que, apesar de o presente estudo apresentar limitações, tais como a possibilidade da ocorrência dos vieses de memória e de causalidade reversa, bem como ter sido realizado em apenas uma UBS do município do Rio de Janeiro, como o desenho de estudo foi apropriado ao que se propunha e como o percentual de perdas não foi expressivo, considera-se que amostra pesquisada foi adequada para estimar os padrões de consumo de álcool bem como seus fatores associados na população atendida pelo serviço de $\mathrm{AB}$ onde foi realizado.

\section{Colaboradores}

RT Jomar coletou e analisou os dados, redigiu e organizou o artigo. AMM Abreu coordenou a pesquisa e revisou artigo. RH Griep colaborou na metodologia, análise estatística e revisou o artigo. 


\section{Referências}

1. World Health Organization (WHO). WHO Expert Committee on problems related to alcohol consumption. $2^{\text {nd }}$ Edition. Geneva: WHO; 2007.

2. World Health Organization (WHO). Global status report on alcohol and health. Geneva: WHO; 2011.

3. Fonseca AM, Galduróz JCF, Noto AR, Carlini ELA Comparison between two household surveys on psychotropic drug use in Brazil: 2001 and 2004. Cien Saude Colet 2010; 15(3):663-670.

4. Moura EC, Malta DC. Consumo de bebidas alcoólicas na população adulta brasileira: características sociodemográficas e tendência. Rev Bras Epidemiol 2011; 14(Supl. 1):61-70.

5. Laranjeira R, Pinsky I, Sanches M, Zaleski M, Caetano R. Alcohol use patterns among brazilian adults. Rev Bras Psiquiatr 2010; 32(3):231-241.

6. Babor TF, Higgins-Biddle JC, Saunders JB, Monteiro MG. The Alcohol Use Disorders Identification Test: Guideline for use in Primary Care. $2^{\text {nd }}$ Edition. Geneva: World Health Organization (WHO); 2001.

7. Magnabosco MB, Formigoni MLOS, Ronzani TM. Avaliação dos padrões de uso de álcool em usuários de serviços de atenção primária à saúde de Juiz de Fora e Rio Pomba (MG). Rev Bras Epidemiol 2007; 10(4):637-647.

8. Cordeiro Q, Michelon L, Ribeiro RB, Kamitsuji C, Silveira CM, Andrade LHG. Triagem para a identificação de uso nocivo de álcool na atenção primária à saúde. Rev Assoc Med Bras 2006; 52(4):200.

9. Minto EC, Corradi-Webster CM, Gorayeb R, Laprega MR, Furtado EF. Intervenções breves para o uso abusivo de álcool em atenção primária. Epidemiol Serv Saúde 2007; 16(3):207-220.

10. Vargas D, Oliveira MAF, Araújo EC. Prevalência da dependência alcoólica em serviços de atenção primária à saúde de Bebedouro, São Paulo, Brasil. Cad Saude Publica 2009; 25(8):1711-1720.

11. Abreu AMM, Jomar RT, Souza MHN, Guimarães RM. Consumo nocivo de bebidas alcoólicas entre usuários de uma unidade de saúde da família. Acta Paul Enferm 2012; 25(2):291-295.

12. Lima CT, Freire ACC, Silva APB, Teixeira RM, Farrel M, Prince M. Concurrent and construct validity of the AUDIT in a urban Brazilian sample. Alcohol Alcohol 2005; 40(6):584-589.

13. Kypri K, McGee R, Saunders JB, Langley JD, Dean JI. Interpretation of items in the AUDIT Questionnaire. Alcohol Alcohol 2002; 37(5):465-467.
14. Ferreira LN, Sales ZN, Casotti CA, Bispo Júnior JP, Braga Júnior ACR. Perfil do consumo de bebidas alcoólicas e fatores associados em um município do Nordeste do Brasil. Cad Saude Publica 2011; 27(8):1473-1486.

15. Almeida LM, Coutinho ESF. Prevalência do consumo de bebidas alcoólicas e de alcoolismo em uma região metropolitana do Brasil. Rev Saude Publica 1993; 27(1):23-29.

16. Barros MBA, Botega NJ, Dalgalarrondo P, MarínLeon L, Oliveira HB. Prevalence of alcohol abuse and factors associated factors in a population-based study. Rev Saude Publica 2007; 41(4):502-509.

17. Amato TC, Silveira OS, Oliveira JS, Ronzani TM. Uso de bebida alcoólica, religião e outras características sociodemográficas em pacientes da Atenção Primária à Saúde - Juiz de Fora, MG, Brasil - 2006. SMAD, Rev Eletrônica Saúde Mental Álcool Drog (Ed. Port.) [periódico na Internet] 2008 jul-dez [acessado 2012 jul 7]; 4:[cerca de 17 p.]. Disponível em http://www2.eerp.udp.br/resmad/artigos.asp

18. Almeida-Filho N, Lessa I, Magalhães L, Araújo MJ, Aquino E, Kawachi I, James SA. Alcohol drinking patterns by gender, ethnicity, and social class in Bahia, Brazil. Rev Saude Publica 2004; 38(1):45-54.

19. Bortoluzzi MC, Traebert J, Loguercio A, Kehrig RT. Prevalência e perfil dos usuários de álcool de população adulta em cidade do Sul do Brasil. Cien Saude Colet 2010; 15(3):679-685.

20. Guimarães VV, Florindo AA, Stopa SR, César CLG, Barros MBA, Carandina L, Goldbaum M. Consumo abusivo e dependência de álcool em populacão adulta no Estado de São Paulo, Brasil. Rev Bras Epidemiol 2010; 13(2):314-325.

21. Primo NLNP, Stein AT. Prevalência do abuso e da dependência de álcool em Rio Grande (RS): um estudo de base populacional. $R$ Psiquiatr RS 2004; 26(3):280-286

22. Laranjeira R, Pinsky I, Zaleski M, Caetanno R, organizadores. I Levantamento Nacional sobre os Padrões de Consumo de Álcool na População Brasileira. Brasília: SENAD; 2007.

23. Costa JSD, Silveira MF, Gazalle FK, Oliveira SS, Hallal PC, Menezes AMB, Gigante DP, Olinto MT, Macedo S. Heavy alcohol consumption and associated factors: a population-based study. Rev Saude Publica 2004; 38(2):284-291. 
24. Rego RA, Oliveira ZMA, Berardo FAN, Oliveira MB, Ramos LR. Epidemiologia do alcoolismo e prevalência da positividade do teste CAGE em inquérito domiciliar no município de São Paulo. Rev ABP-APAL 1991; 13(2):75-90.

25. Buss PM. Promoção da saúde e qualidade de vida. Cien Saude Colet 2000; 5(1):163-177.

26. Lima-Costa MF. Influência da idade e da escolaridade no uso de serviços preventivos de saúde Inquérito de Saúde da Região Metropolitana de Belo Horizonte, Minas Gerais, Brasil. Epidemiol Serv Saúde 2004; 13(4):201-208.

27. Bastos FI, Bertoni N, Hacker MA. Consumo de álcool e drogas: principais achados de pesquisa de âmbito nacional, Brasil 2005. Rev Saude Publica 2008 (Supl. 1); 42:109-117.

28. Souza EHA, Oliveira PAP, Paegle AC, Goes PSA. Raça e o uso dos serviços de saúde bucal por idosos. Cien Saude Colet 2012; 17(8):2063-2070.

29. Brasil. Ministério da Saúde (MS). Política Nacional de Saúde Integral da População Negra. Brasília: MS; 2007.

30. Jhingan HP, Shyangwa P, Sharma A, Prasad KM, Khandelwl SK. Prevalence of alcohol dependence in a town in Nepal as assessed by the CAGE questionnaire. Addiction 2003; 98(3):339-343.

31. Malyutina S, Bobak M, Kurilovitch D, Nikitin Y, Marmot M. Trends in alcohol intake by education and marital status in urban population in Russia between the mid 1980s and the 1990s. Alcohol Alcohol 2004; 39(1):64-69.

32. Wild TC, Roberts AB, Cunningham J, Schopflocher D, Pzaderka-Robinson H. Alcohol problems and interest in self-help: a population study of Alberta adults. Can J Public Health 2004; 95(2):127-132.

33. Karlamangla A, Zhou K, Reuben D, Greendale G, Moore A. Longitudinal trajectories of heavy drinking in adults in the United States of America. Addiction 2006; 101(1):91-99.

34. Blue I. Individual and contextual effects on mental health status in São Paulo, Brazil. Rev Bras Psiquiatr 2000; 22(3):116-123.

35. Domingos JBC, Pillon SC. O uso de álcool entre motoristas no interior do Estado de São Paulo. Rev enferm UERJ 2007; 18(3):377-382.

36. Volcan SMA, Sousa PLR, Mari JJ, Horta BL. Relação entre bem-estar espiritual e transtornos psiquiátricos menores: estudo transversal. Rev Saude Publica 2003; 37(4):404-415.
37. Berto SJP, Carvalhaes MABL, Moura EC. Tabagismo associado a outros fatores comportamentais de risco de doenças e agravos crônicos não transmissíveis. Cad Saude Publica 2010; 26(8):1573-1582.

38. Galán I, Rodriguez-Artalejo F, Tobías A, Díez-Gañán L, Gandarilhas A, Zorrilla B. Agregácion de factores de riesgo ligados al comportamiento y su relación com la salud subjetiva. Gac Sanit 2005; 19(5):1-9.

39. Poortinga W. The prevalence and clustering of four lifestyle risk factors in an English adult population. Prev Med 2007; 44(2):124-128.

40. Instituto Nacional de Câncer (INCA). Inquérito domiciliar sobre comportamentos de risco e morbidade referida de doenças e agravos não transmissíveis em 15 capitais brasileiras e no Distrito Federal 2002-2003. Rio de Janeiro: INCA; 2005.

41. Lima-Costa MF, Firmo JOA, Uchôa E. A estrutura da auto-avaliação da saúde entre idosos. Projeto Bambuí. Rev Saude Publica 2004; 38(6):827-834.

42. World Health Organization (WHO). Preventing Chronic Diseases a vital investment. Geneva: WHO; 2005.

43. Meloni JN, Laranjeira R. Custo social e de saúde do consumo do álcool. Rev Bras Psiquiatr 2004; 28(Supl. 1):S7-S10.

44. Corrao G, Bagnardi B, Zambon A, La Vechia C. A meta-analysis of alcohol consumption and the risk of diseases. Prev Med 2004; 38(5):613-619.

45. Fontanella BJB, Demarzo MMP, Mello GA, Fortes SLCL. Os usuários de álcool, atenção primária à saúde e o que é “perdido na tradução". Interface (Botucatu) 2011; 15(1):573-585.

46. McQuade WA, Levy SM, Yanek LR, Davis SW, Liepman MR. Detecting symptoms of alcohol abuse in primary care settings. Arch Fam Med 2000; 9(9):814821.

47. Jomar RT, Paixão LAR, Abreu AMM. Alcohol Use Disorders Identification Test (AUDIT) e sua aplicabilidade na atenção primária à saúde. Rev APS 2012; 15(1):113-117.

Artigo apresentado em 29/08/2012

Aprovado em 15/10/2012

Versão final apresentada em 31/10/2012 
